\section{Some Comments On Providing Broadband Light To Users}

\author{
S. Benson, \\ Thomas Jefferson National Accelerator Facility., Newport News VA USA
}

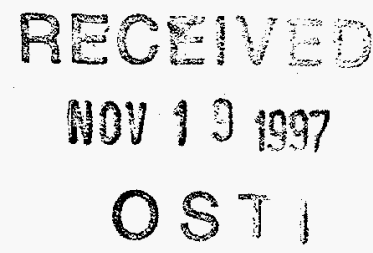

Abstract

Though the free-electron laser gain medium is inherently broadband and several labs have demonstrated wavelength tuning over a range of several octaves, it is quite challenging to deliver such a broad bandwidth to users. The challenge arises from providing high quality, high power light, transporting the light to the users, and providing diagnostics to the user over a very large wavelength range. This paper summarizes some of the issues which must be considered and discusses some of the solutions which user facilities around the world have used to address the problems.

\section{Introduction}

There are now many FEL user facilities worldwide and several provide broadband tunability. In this paper, I describe the problems researchers have had taking advantage of broadband tunability and some of the solutions they have adopted.

The resonant wavelength of the free-electron laser is given by the equation

$$
\lambda_{0}=\frac{\lambda_{\mathrm{rr}}}{2 h \gamma^{2}}\left[1+K^{2}+(\gamma \theta)^{2}\right]
$$

where $h$ is the harmonic number for harmonic lasing, $\gamma$ is the electron beam relativistic energy divided by its rest mass, $\theta$ is the mean relative angle between the electron beam and the optical mode, and $K$ is the wiggler parameter given by $0.934 \lambda_{r r} B$ where $B$ is the rms wiggler field in Tesla and $\lambda_{w}$ is the wiggler wavelength in $\mathrm{cm}$. One can tune using each or all of these parameters. Changing the wiggler wavelength is done by using multiple wigglers. Tuning using the mean angle has never been demonstrated and only third harmonic lasing has been demonstrated to date [1].

From a user standpoint, there are two important issues in tuning the laser:

1.) Ease of Tuning. The user (and the data acquisition computer) should have full control of laser tuning over a large wavelength range ( $\pm 20 \%$ for example).

2.) Uniform output power and beam quality. The power, spectral bandwidth, stability, and transverse mode quality should change little over the available scanning range. Changes in these parameters can mask true wavelength dependence in the sample being studied.

Though, in principle, energy tuning can meet these demands, the most convenient and uniform tuning performance to date has been using wiggler field tuning. The FELIX lasers in FOM [2], the CLIO laser in Orsay[3], the
Vanderbilt and Duke lasers in the U.S. $[4,5]$, and the Firefly laser at Stanford [6] all tune over one octave of frequency by wiggler tuning alone.

In order to make the tuning range as large as possible it is best to make the maximum wiggler parameter $K$ as large as possible. As an example, a proposed IRFEL at Jefferson Lab can tune over a factor of ten (from 2.4 to 24 $\mu \mathrm{m}$ at $100 \mathrm{MeV}$ ), with little change in the laser gain, by having a maximum rms $K$ of 4 .

For low electron beam energy one is forced to operate with a low $K$ wiggler and thus must tune using energy. Several user facilities have used this approach and have shown that tuning over a wavelength range of several percent is easy and tuning over $\pm 20 \%$ is possible using computer control. If one tunes over more than a factor of 2 , the power variation with wavelength starts to become substantial, This problem can be overcome using multiple wigglers as is done at UCSB. [7]

\section{Optics}

Several features in the optical system may limit tunability. Even if the gain versus wavelength is constant, the power versus wavelength will vary unless the laser gainto-loss ratio stays in the range of 3 to 5 and the electron beam energy is fixed. In order to keep the power relatively constant versus wavelength it is best to tune by changing the wiggler strength so that the electron beam energy and the laser gain are approximately constant, have a total cavity loss which is in the range of $1 / 3$ to $1 / 5$ of the small signal gain independent of wavelength, and have a ratio of output coupling to total losses (coupling efficiency) which is independent of wavelength.

\subsection{Diffraction:}

Power at long wavelengths may fall off due to diffraction in the wiggler bore. Diffraction reduces both the out- 


\section{DISCLAIMER}

This report was prepared as an account of work sponsored by an agency of the United States Government. Neither the United States Government nor any agency thereof, nor any of their employees, make any warranty, express or implied, or assumes any legal liability or resporsibility for the accuracy, completeness, or usefulness of any information, apparatus, product, or process disclosed, or represents that its use would not infringe privately owned rights. Reference herein to any specific commercial product, process, or service by trade name, trademark, manufacturer, or otherwise does not necessarily constitute or imply its endorsement, recommendation, or favoring by the United States Government or any agency thereof. The views and opinions of authors expressed herein do not necessarily state or reflect those of the United States Government or any agency thereof. 


\section{DISCLADIER}

Portions of this doeconent mey be illegible in electronic imsge prodnets. Insages are produced from the best available original docoment 
put coupling efficiency and the laser gain (via mode distortion). The obvious solution is to increase the wiggler gap (and the wiggler period to compensate the decreased field). When there is only room for one wiggler in the accelerator vault, it is best to design the wiggler to accommodate the longest desirable wavelength and get to shorter wavelengths via harmonic generation or harmonic lasing, provided the normalized electron beam emittance $\varepsilon_{n}<\gamma \lambda / 4 \pi$.

\subsection{Output coupling and absorption}

At short wavelengths the gain generally falls and the absorption and scatter rise. This problem is sometimes alleviated using dielectric mirrors. For $\lambda<1 \mu \mathrm{m}$, dielectric mirrors (with a $20 \%$ bandwidth) must be used in any case. A mirror turntable, demonstrated at FELI in Osaka [8] can make tuning at short wavelengths quite convenient.

From a broadband user standpoint, the best method of output coupling at wavelengths longer than $4 \mu \mathrm{m}$ is hole coupling. It allows very broadband operation with a cavity length independent of wavelength. The change in the output coupling efficiency versus wavelength can be minimized by using a variable aperture in the cavity as shown by Xie [9]. The main disadvantages to hole coupling are a low damage threshold and an intrinsically low output coupling efficiency due to scatter from the hole edge. A mirror turntable might be used in a hole-coupled cavity to better optimize the output coupling versus wavelength.

The UCSB lasers and the Firefly laser at Stanford have used an insertable scraper mirror in a stable resonator. This allows easy adjustment of the output coupling but good measurements of the output coupling efficiency have not been done to date and the mode quality can be poor.

\subsection{Beam transport}

Due to strong atmospheric absorption in the infrared, FELs must have a purged or evacuated transport line. For long runs, an evacuated beam transport is required.

Since metal optics are used, the losses in the infrared, even for a complicated system, can be less than $20 \%$. For wavelengths shorter than $1 \mu \mathrm{m}$, one must use dielectric mirrors to reduce losses. Broadband coatings are available which cover a $40 \%$ or greater wavelength range. Since it is tedious to change out all the coatings in a long evacuated transport line, the ease of tuning in a visible or UV FEL will be poor. Runs in a given wavelength band must be scheduled when the proper optics are installed.

One option which allows several wavelength to be used is to use multiple beam transport lines with optical switchyards to allow different labs access to each wavelength range. This has been accomplished at both UCSB [7] and FELI [8].

\section{Diagnostics}

A user or operator must be able to measure the power versus time, wavelength, and transverse position, both at saturation and at start-up. For millisecond to nanosecond pulses, measurements of the saturated laser output can be made with pyroelectric detectors. These are extremely broadband and can be used over almost the entire electromagnetic spectrum. One and two dimensional arrays are available for mode structure and spectrum. Continuous power can be measured using a thermopile detector.

For the fastest time scales and spontaneous power detection, the pyroelectric detector is not sufficiently sensitive to be useful. Cooled $\mathrm{HgCdTe}, \mathrm{CuGe}$, and $\mathrm{HgGe}$ can be used for these applications, though they can only cover an octave or two in wavelength range apiece.

To measure picosecond pulses, the best technique is to use either an autocorrelation measurement. An autocorrelator must have a harmonic rejection filter both on the input and the output, a beam splitter, and a doubling crystal. All of these tend to be strongly wavelength dependent. A wavelength range of 8 to $32 \mu \mathrm{m}$ has been measured using a device built by Knippels at FELIX. Material properties at wavelengths longer than $32 \mu \mathrm{m}$ make the operation of an autocorrelator extremely difficult. One possible solution to this problem is to use sum frequency mixing of a conventional near infrared laser with FEL light on a surface [10]. This produces a pulse of light at a visible or near-infrared wavelength which can be analyzed using conventional diagnostics.

\section{Conclusions}

FELs have already shown enormous tunability in many facilities around the world. I have tried to present some examples here of how people have taken advantage of this extremely broadband gain medium and some other ideas which might be tried to make better use of the lasers we now have.

Acknowledgments: This work was supported DOE Contract \#DE-AC05-84ER40150 and the Laser Processing Consortium.

\section{References}

[1] S. V. Benson and J. M. J. Madey, Phys. Rev. A 39 (1989) 1579.

[2] D. Oepts, A.F. G. van der Meer, and P. W. Amersfoort, Inf. Phys. Tech. 36 (1995) 297.

[3] J. M. Ortega et al., Nucl. Inst. and Meth. A 375 (1996) 618.

[4] C. A. Brau, Nucl. Inst. and Meth. A 31 (1992) 38.

[5] G. A. Barnett, J. M. J. Madey, C. B. McGee, K. D. Straub, and E. B. Szarmes, Nucl. Inst. and Meth.. A 375 (1996) 97.

[6] K. W. Berryman and T. I. Smith, Nucl. Inst. and Meth.. A 375 (1996) 6.

[7] G. Ramian, Nucl. Inst. and Meth.. A 318 (1992) 225.

[8] S. Okuma et al., Nucl. Inst. and Meth.. A 375 (1996) 654.

[9] M. Xie and K. J. Kim, Nucl. Inst. and Meth.. A 318 (1992) 877.

[10] F. Glotin et al., Nucl. Inst. and Meth.. A 375 (1996) 558. 